\title{
Full Current Statistics in the Regime of Weak Coulomb Interaction
}

\author{
D. A. Bagrets and Yu. V. Nazarov \\ Department of Applied Physics and Delft Institute of Microelectronics and Submicrontechnology, \\ Delft University of Technology, Lorentzweg 1, 2628 CJ Delft, The Netherlands
}

(Dated: February 2, 2008)

\begin{abstract}
We evaluate the full statistics of the current via a Coulomb island that is strongly coupled to the leads. This strong coupling weakens Coulomb interaction. We show that in this case the effects of the interaction can be incorporated into the renormalization of transmission eigenvalues of the scatterers that connect the island and the leads. We evaluate the Coulomb blockade gap in the current-voltage characteristics, the value of the gap being exponentially suppressed as compared to the classical charging energy of the island.
\end{abstract}

PACS numbers: 73.23.-b, 73.23.Hk, 72.70.+m, 05.40.-a

The electric charge is quantized. There are two important manifestations of this fact in electron transport on mesoscopic scale.

The first manifestation is a shot noise [1]. The quantum mechanics modifies the shot noise in an arbitrary mesoscopic conductor with respect to its classical value $S=2 e I$. It also changes the full statistics of charge transfer via a mesoscopic scatter, so that the statistics is not the simple Poissonian one [2].

The second manifestation of electron charge quantization is the Coulomb blockade phenomenon. The Coulomb blockade is most strong provided the conductance $G$ of a mesoscopic system is much smaller than the conductance quantum $G_{Q}=e^{2} / 2 \pi \hbar[\underline{3}]$. The statistics of charge transfer in this strongly interacting case is that of a classical stochastic Markov process [4]. On the contrary, quantum mechanics is of importance for Coulomb blockade in the opposite limit $G \gg G_{Q}$ where Coulomb interaction is weakened.

The charge quantization manifests itself in both ways in full current statistics under conditions of weak Coulomb interaction, this provides the motivation for the present study.

It has been understood that the charge quantization survives even in the limit $G \gg G_{Q}$ [5, 6, 7, 8, 9, 10]. The ground state energy of the Coulomb island was shown to retain the periodic dependence on the induced "off-set" charge $q$, thus indicating the Coulomb blockade. However, due to large quantum fluctuations of charge in the island, the effective charging energy $\widetilde{E}_{C}$ (defined as the periodic $q$-dependent part of free energy) is exponentially suppressed as compared to the classical charging energy $E_{C}=e^{2} / 2 C$. It is important that the weak charge quantization persists not only for tunnel junctions [5, 6, 7] connecting the island and the leads. The connectors can be arbitrary mesoscopic scatterrers [10]. The quantization completely vanishes only for constrictions with perfectly transmitting channels [8, [9].

This research was concentrated on the equilibrium properties, an alternative is to study transport. Recent studies link the short noise in the conductor to the inter- action correction to the conductance. 11] The interaction correction to full current statistics was analyzed in 12. for a scatterer embedded in the electromagnetic environment. The relation between interaction correction to the conductance and the formation of Coulomb blockade in an island was investigated in 13 .

In this Letter we evaluate the full statistics of the current via the Coulomb island defined by several mesoscopic scatterers assuming weak Coulomb interaction, $G \gg G_{Q}$. The results can be summarized as follows. At energy scale $E \ll g_{0} E_{c}, g_{0}=G_{0} / G_{Q}$ being the dimensionless conductance of the system in the absence of interaction, the dominant effect of Coulomb interaction is the energy-dependent renormalization of the transmission eigenvalues $T_{n}^{[k]}$ of the mesoscopic scatterers labeled by $k$,

$$
\frac{d T_{n}^{[k]}}{d \ln E}=\frac{2 T_{n}^{[k]}\left(1-T_{n}^{[k]}\right)}{\sum_{n, k} T_{n}^{[k]}} .
$$

The renormalization of a similar form was previously obtained in [18] for a scatteter in the weakly interacting 1d gas and in [12] for a single multi-channel scatterer shunted by an external impedance. We thus prove this simple relation for a Coulomb island. The full current statistics is readily obtained from the energy-dependent $T_{n}^{[k]}$ with using non-interacting scattering theory approach of 2, 15]. This gives the voltage dependence of conductance, shot noise and all higher cumulants of charge transfer. In contrast to the case of a single scatterer, the renormalization of all transmission eigenvalues may break down at finite energy $\widetilde{E}_{C} \propto g_{0} E_{C} e^{-\alpha g_{0}}$, $\alpha$ being a numerical factor depending on the details of the initial transmission distribution. Remarkably, $\widetilde{E}_{C}$ coincides with the equilibrium effective charging energy evaluated with instanton technique. 10] However, the renormalization stops at the effective Thouless energy $E_{\mathrm{Th}} \sim G(E) \delta / G_{Q}, \delta$ being mean level spacing in the island. This gives rise to two distinct scenarios at low enegy. If $g_{0}>\alpha^{-1} \ln \left(E_{C} / \delta\right)$, Coulomb blockade does not occur with zero-bias conductance being saturated at 


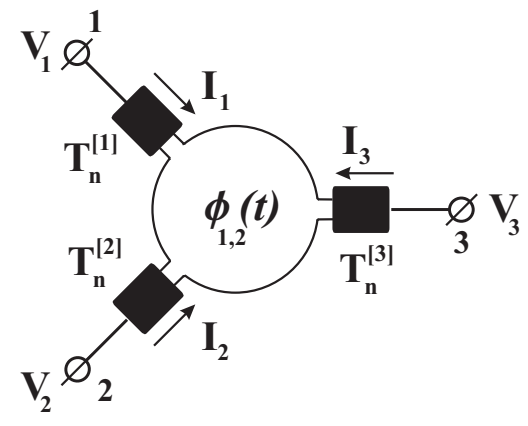

FIG. 1: Multi-terminal ( $\mathrm{M}=3)$ Coulomb island.

the value $G\left(E_{\mathrm{Th}}\right) \gg G_{Q}$. Alternatively, $G(0) \approx 0$ and $\widetilde{E}_{C}$ defines the Coulomb gap.

Let us give the details of the model in use. The Coulomb island is characterized by two parameters: charging energy $E_{C}$ and the mean level spacing $\delta, E_{C} \gg$ $\delta$. The island is connected to $M \geq 2$ external leads by means of $M$ arbitrary mesoscopic scatterers. (Fig. 1) Each scatterer, or connector, $i$ is characterized by the set of transmission eigenvalues $T_{n}^{[i]}$. We assume that the island is strongly coupled to the leads, $g_{0}=\sum_{n, m} T_{n}^{[i]} \gg 1$. Our goal is to evaluate the cumulant generating function (CGF) $S\left(\left[\chi_{i}\right]\right)$ ). The Fourier transform of $\exp (-S)$ with respect to counting fields $\chi_{i}$ gives the probability $P\left(\left\{N_{i}\right\}\right)$ for $N_{i} \gg 1$ electrons to be transferred to the terminal $i$ during time interval $t_{0}$. (See 2] ) The derivatives of $S$ give the average value of currents, shot-noise correlations and higher order moments of charge transfer.

To evaluate the CGF of the Coulomb island, we have extended the semiclassical approach for the FCS of the non-interacting electrons [15]. To account for Coulomb interactions, we introduce a dynamical phase variable $\varphi(t)$ [14] that results from the Hubbard-Stratonovich transform of the charging energy. Its time derivative, $\dot{\varphi}(t) / e$, presents the fluctuating electrostatic potential of the island. The CGF $S\left(\left\{\chi_{i}\right\}\right)$ can be then represented in the form of a real-time path integral over the fields $\varphi_{1,2}(t)$ residing at two branches of the Keldysh contour

$$
\begin{aligned}
& \exp \left(-S\left\{\chi_{i}\right\}\right)=\int D \varphi_{1,2}(t) \exp \left\{\frac{i}{2} E_{C}^{-1} \int_{-\infty}^{+\infty} d t\left(\dot{\varphi}_{1}^{2}-\dot{\varphi}_{2}^{2}\right)\right. \\
& \left.-\sum_{k} S_{\operatorname{con}}^{[k]}\left(\left\{\hat{G}, \hat{G}_{k}^{\chi}\right\}\right)-i \pi \delta^{-1} \operatorname{Tr}\left\{\left(i \partial_{t}-\dot{\Phi}\right) \hat{G}\right\}\right\}
\end{aligned}
$$

Here $\hat{\Phi}=\left(\begin{array}{cc}\phi_{1}(t) & 0 \\ 0 & \phi_{2}(t)\end{array}\right)$ is the matrix in Keldysh space, $2 \times 2$ matrix $\hat{G}\left(t_{1}, t_{2}\right)$ presents the electron Green function in the island that implicitly depends on $\varphi_{1,2}(t)$. The trace operation includes the summation over Keldysh indices and the integration in time. The contribution of each connector $S_{\text {con }}^{[k]}$ has a form found in the circuit theory [15, 16]

$$
S_{\text {con }}^{[k]}=-\frac{1}{2} \sum_{n} \operatorname{Tr} \ln \left[1+\frac{1}{4} T_{n}^{[k]}\left(\left\{\hat{G}, \hat{G}_{k}^{\chi}\right\}-2\right)\right]
$$

$\left\{\hat{G}, \hat{G}_{k}^{\chi}\right\}$ denoting the anticommutator of the Green functions with respect to both Keldysh and time indices. The Green functions in the leads $\hat{G}_{k}(\chi)$ are obtained by $\chi$-dependent gauge transformation [16] of the equilibrium Green functions in the reservoir $k, \hat{G}_{k}^{[0]}, \hat{G}_{k}^{\chi}(\epsilon)=$ $\exp \left(i \chi_{k} \bar{\tau}_{3} / 2\right) \hat{G}_{k}^{(0)}(\epsilon) \exp \left(-i \chi_{k} \bar{\tau}_{3} / 2\right)$, where $\hat{G}_{k}^{[0]}$ are given by $\bar{G}_{k}^{[0]}=\left(\begin{array}{cc}1-2 f_{k} & -2 f_{k} \\ -2\left(1-f_{k}\right) & 2 f_{k}-1\end{array}\right)$. Here $f_{k}(\varepsilon)$ presents the electron distribution function in the $k$-th reservoir. The expression (3) is valid under assumption of instantaneous electron transfer via a connector, this corresponds to energy independent $T_{n}^{[k]}$.

In order to find $\hat{G}\left(t_{1}, t_{2}\right)$ at given $\varphi_{1,2}(t)$, we minimize the action with respect to all $\hat{G}\left(t_{1}, t_{2}\right)$ subject to the constrain $\hat{G} \circ \hat{G}=\delta\left(t_{1}-t_{2}\right)$. This yields the saddle point equation for $\hat{G}\left(t_{1}, t_{2}\right)$ :

$$
\sum_{n, k} \frac{T_{n}^{[k]}\left[\hat{G}_{k}^{\chi}, \hat{G}\right]}{4+T_{n}^{[k]}\left(\left\{\hat{G}_{k}^{\chi}, \hat{G}\right\}-2\right)}=i \pi \delta^{-1}\left[i \partial_{t}-\dot{\Phi}, \hat{G}\right]
$$

where $[. ., .$.$] denotes the commutator in the Keldysh-$ time space. This relation expresses $\hat{G}\left(t_{1}, t_{2}\right) \equiv$ $\hat{G}\left(t_{1}, t_{2} ;\left[\varphi_{1,2}(t)\right]\right)$ via the reservoir Green functions $\hat{G}^{[k]}$. This circuit theory relation is similar to obtained in [15]. It disregards the mesoscopic fluctuations, since those lead to corrections of the order of $\sim 1 / g_{0}$ at all energies, whereas the interaction corrections are of the order of $\sim 1 / g_{0} \ln (E)$ tending to diverge at small energies. If $\varphi_{1,2}(t)=0$, Eq. (4) separates in energy representation and coincides with that of Ref. 15].

This sets the model. We start the analysis of the model with perturbation theory in $\varphi_{1,2}$ around the semiclassical saddle point $\hat{G}\left(t_{1}, t_{2}\right)=\hat{G}_{0}, \varphi_{1,2}(t)=0$. The phase fluctuations are small, $\delta \varphi^{2} \sim 1 / g_{0}$, so we keep only quadratic terms to the action (2). The resulting Gaussian path integral over $\varphi_{1,2}$ can be readily done. This procedure is equivalent to the summation of all one-loop diagrams of the conventional perturbation theory, i.e. to the "random-phase approximation" (RPA).

For the rest, we restrict ourselves to the most interesting low voltage/temperature limit, $\max \{e V, k T\} \ll$ $g_{0} E_{C}$. In this limit, we evaluate the interaction correction to the CGF with the logarithmic accuracy. It reads

$$
\begin{aligned}
& \Delta S_{\chi}=\frac{t_{0}}{g_{0}} \ln \left(\frac{g_{0} E_{C}}{\max \{e V, k T\}}\right) \times \\
& \int \frac{d \varepsilon}{2 \pi} \sum_{n, k} \frac{2 T_{n}^{[k]}\left(1-T_{n}^{[k]}\right)\left(\left\{\hat{G}_{k}^{\chi}, \hat{G}_{0}\right\}-2\right)}{4+T_{n}^{[k]}\left(\left\{\hat{G}_{k}^{\chi}, \hat{G}_{0}\right\}-2\right)}
\end{aligned}
$$

provided $\max \{e V, k T\}>E_{\mathrm{Th}}$, where $E_{\mathrm{Th}}=g_{0} \delta$ is the Thouless energy of the island. In the opposite case, 
$\max \{e V, k T\}<E_{\mathrm{Th}}$, the voltage/temperature should be replaced with $E_{\mathrm{Th}}$. Note, that the correction (5) is contributed by only virtual inelastic processes that change the probabilities of real elastic scatterings.

For simplicity, we consider the shot-noise limit $e V \gg$ $k T$ only. Then the magnitude of the correction shall be compared with the zero-order CGF $S_{\chi}^{[0]} \sim t_{0} e V g_{0}$. This implies that the perturbative RPA result (5) is applicable only if $g_{0}^{-1} \ln \left(g_{0} E_{C} / e V\right) \ll 1$. At lower voltages $\Delta S_{\chi}$ logarithmically diverges. This indicates that we should proceed with a renormalization group (RG) analysis.

We perform the RG analysis of the action (2) in the one-loop approximation, this corresponds to the "poor's man scaling". This is justified by $g \gg 1$. We follow the conventional procedure [19] and decompose $\varphi(t)$ onto the fast $\varphi_{f}$ and slow parts $\varphi_{s}$. On each step of RG procedure we eliminate the fast degrees of freedom in the energy range $E-\delta E<\omega<E$ to obtain new action $S_{E-\delta E}\left[\varphi_{s}\right], E$ being the current ultraviolet cutoff. Our key result is that the change in the action at each step of RG procedure can be presented as a change of transmission eigenvalues $T_{n}^{[k]}$.

Therefore, the RG equations can be written directly for transmission eigenvalues and take a simple form (11). The equations are to be solved with initial conditions at the upper cutoff energy $E=g_{0} E_{C}$, those are given by "bare" transmission eigenvalues $T_{n}^{[k]}\left(E=g_{0} E_{C}\right)=$ $T_{n}^{[k]}$. The RG equations resemble those for the transmission coefficient for a scatterer in the weakly interacting one-dimensional electron gas [18] and for a single multi-channel scatterer in the electromagnetic environment 12]. The effective impedance $Z$ is just replaced by inverse conductance of the island to all reservoirs, $G(E)=G_{Q} \sum_{n, k} T_{n}^{[k]}(E)$. The important difference is that this conductance is itself subject to renormalization. The difference becomes most evident in the case when all contacts are tunnel junctions, $T_{n}^{[k]} \ll 1$. In this case, one can sum up over $k, n$ in Eqs. (1) to obtain the RG for the conductance only : $d G / d \ln E=2 G_{Q}$. This renormalization law 20] was recently applied to conductance of granular metals. The Eqs. (11) could be also derived in the framework of functional RG approach to $\sigma$-model of disordered metal. 21].

We solve the RG Eqs. (1) in general case to obtain

$$
\begin{aligned}
T_{n}^{[k]}(E) & =T_{n}^{[k]} y /\left(1-T_{n}^{[k]}(1-y)\right), \\
\ln \left(g_{0} E_{C} / E\right) & =-\frac{1}{2} \sum_{n, k} \ln \left(1-T_{n}^{[k]}(1-y)\right)
\end{aligned}
$$

The first equation gives the renormalized transmission eigenvalues at a given value $E$ of the upper cutoff in terms of variable $y(E), 0 \leq y \leq 1$. The second equation implicitly expresses $y(E)$.

We note that the energy dependence of transmission coefficients induced by interaction is very weak provided

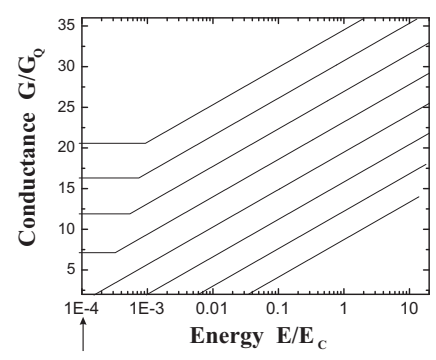

(a)

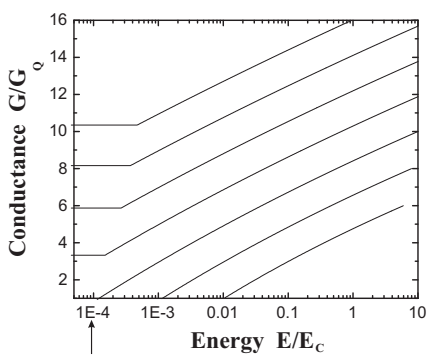

(b)
FIG. 2: The total conductance of the Coulomb island versus the energy: two scenarios. We assume $\ln \left(E_{c} / \delta\right)=10.0$. Arrows show the energy scale $\sim \delta$. Pane (a): tunnel connectors, $g_{0}$ changes from 42 (upper curve) to 14 (lowermost curve) with the step 4 . Pane (b): diffusive connectors, $g_{0}$ changes from 18 to 6 with the step 2 .

$G(E) \gg G_{Q}$ : If energy is changed by a factor of two, the conductance is changed by $\sim G_{Q}$. To use the equations for evaluation of FCS at given voltages $V^{[k]}$ of the leads, one takes $T_{n}^{[k]}(E)$ at upper cutoff $E=\max _{k}\left(V^{[k]}\right)$, and further disregards their energy dependence. Then one can follow the lines of Ref. [15]: It is convenient to introduce the function $S^{[k]}(x)=-\sum_{n} \ln \left[1+\frac{1}{2} T_{n}^{[k]}(x-1)\right]$ to incorporate all required information about transmission eigenvalues. The renormalization of $S^{[k]}$ in terms of $y$ is especially simple: $S^{[k]}(x, y)=S^{[k]}((x+1) y-$ $1)-S^{[k]}(2 y-1)$. From this one readily finds the conductance of each scatterer, $G^{[k]}(y)=2 G_{Q} \partial S^{[k]} / \partial x(1, y)$, as well as the renormalized transmission distribution $T^{2} \rho^{[k]}(T, y)=(2 / \pi) \operatorname{Im}\left\{\partial S^{[k]} / \partial x(1-2 / T-i 0, y)\right\}$.

The RG equations (1) have a fixed point at $T_{n}^{[k]}=$ $0, y=0$ that occur at finite energy

$$
E=\widetilde{E}_{C}=g_{0} E_{C} \prod_{k, n}\left(1-T_{n}^{[k]}\right)^{1 / 2}
$$

This indicates the breakdown of RG and formation of Coulomb blockade with the exponentially small gap $\widetilde{E}_{C}$. The same energy scale was obtained from equilibrium instanton calculation of Ref. [10]. For a field theory, one generally expects different physics and different energy scales for instantons and perturbative RG. The fact that these scales are the same shows a hidden symmetry of the model which is yet to understand. [22]

Alternative low-energy behavior is realized if the current cut-off reaches $E_{\mathrm{Th}}=G(E) \delta / G_{Q}$. (Fig.2) The log renormalization of the transmission eigenvalues stops at this point and their values saturate. We thus predict a sharp crossover between the two alternative scenarios, that occur at value of $g_{0}=g_{c}$ corresponding to $\widetilde{E}_{C} \simeq \delta$. This value equals $g_{c}=\alpha^{-1} \ln \left(E_{C} / \delta\right)$, where $\alpha=\frac{1}{2} g_{0}^{-1} \sum_{n, k} \ln \left(1-T_{n}^{[k]}\right)$, and depends on transmission distribution of all connectors. If all connectors are tunnel juctions, $\alpha_{T}=2$. For diffusive connectors, $\alpha_{D}=\pi^{2} / 8$ and the energy dependence of the total conductivity is 


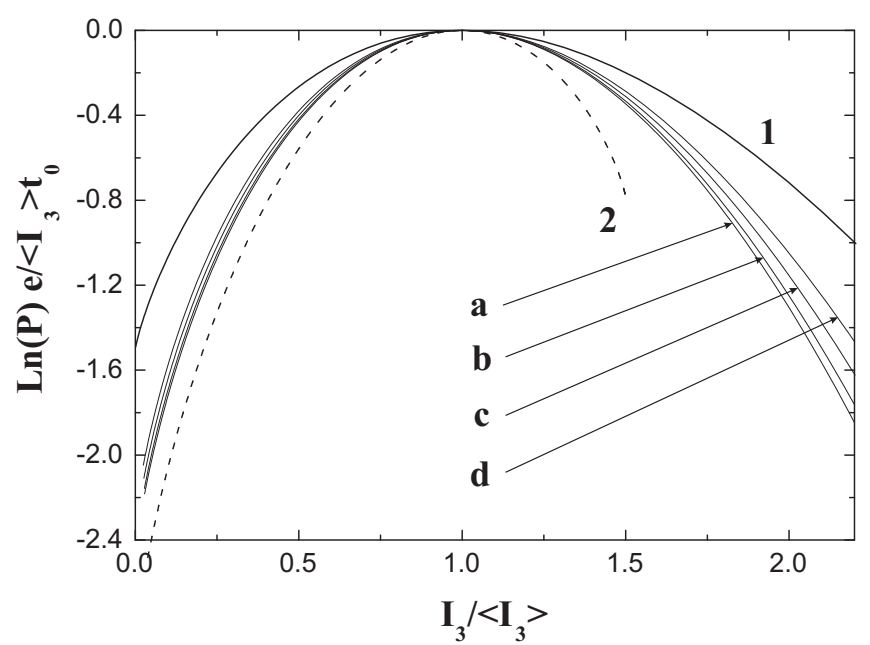

FIG. 3: An example of FCS: Probability to measure equal currents in the 3 -terminal island with identical junctions. The curves (a-d) present diffusive connectors at different values of the renormalization parameter $y(e V)$ : (a) - 1.0, (b) - 0.5, (c) $-0.1,(d)-0$. No interaction effect is seen for tunnel (1) and ballistic (2) connectors.

given by $g_{D}(V) \sim g_{0} \sqrt{\xi} \operatorname{ctg} \sqrt{\xi}, \xi \equiv 2 g_{0}^{-1} \ln \left(g_{0} E_{C} / e V\right)$. (Fig. 2)

To give an example of FCS calculation in the regime of weak Coulomb interaction, we consider the 3 -terminal Coulomb island with identical junctions, discussed in 15 . (Fig. 1). The voltages applied are $V_{3}=V, V_{1,2}=0 \mathrm{We}$ plot in Fig. 3 the $\log (P)$, the logarithm of the probability to measure the same currents to the terminals 1 and 2 , $I_{1}=I_{2}=-I_{3} / 2$, versus the current $I_{3}$ measured in the terminal 3 (Fig. 3). Both $\log (P)$ and $I_{3}$ are normalized by the average current $\left\langle I_{3}\right\rangle$, so that in the absence of interaction the curves corresponding to different voltages are the same (assuming $\mathrm{eV} \gg T$ ), the shape of the curve reflecting the transmission distribution in the contacts. To take interaction into account, we change the transmission eigenvalues according to Eqs. $(6,7)$ and evaluate the probability with the method of Ref. 15].

The curves 1 (tunnel junctions) and 2 (ballistic contacts) stay the same not depending on the renormalization. Indeed, the renormalization does not affect ballistic transmission, as to tunnel junctions, it affects only their conductances. The interaction effect is visible for diffusive junctions. The curves (a)-(d) correspond to different values of $y(E=e V)$. The transmission distribution of each contact evolves from the diffusive form $\left(\rho_{D}(T)=\right.$ $g(y) / 2 T \sqrt{1-T})$ at the highest voltage $(y \approx 1)$ to the double junction form $\left(\rho_{D J}(T)=g(y) / \pi T^{3 / 2} \sqrt{1-T}\right)$ at the lowest voltage $(y \approx 0)[12$. This visibly changes the form of $\log P\left(I_{3}\right)$.

To conclude, we have analyzed the effect of weak Coulomb interaction $\left(G \gg G_{Q}\right)$ on FCS in Coulomb is- land. We have revealed that the interaction effect can be incorporated into a simple energy-dependent renormalization of transmission eigenvalues, this enables easy evaluation of all transport properties. The Coulomb blockade develops only if the total conductance of the island is below some critical value $\sim G_{Q} \log \left(E_{C} / \delta\right)$, otherwise the interaction correction to transport saturates at low energies.

This work is a part of the research program of the "Stichting voor Fundamenteel Onderzoek der Materie" (FOM), and we acknowledge the financial support from the "Nederlandse Organisatie voor Wetenschappelijk Onderzoek" (NWO).

[1] Ya. M. Blanter and M. Büttiker, Phys. Rep. 336, 1 (2000).

[2] L. S. Levitov and G. B. Lesovik, JETP Lett. 58, 230 (1993); L. S. Levitov, H.-W. Lee, and G. B. Lesovik, Journal of Mathematical Physics, 37 (1996) 10.

[3] G.-L. Ingold, Yu. V. Nazarov, in Single Charge Tunneling, NATO ASI Series B: 294, ed. H. Grabert, M. H. Devoret (NewYork, 1992).

[4] D. A. Bagrets and Yu. V. Nazarov, Phys. Rev. B 67, 085316 (2003).

[5] S. V. Panyukov and A. D. Zaikin, Phys. Rev. Lett. 67, 3168 (1991).

[6] X. Wang and H. Grabert, Phys. Rev. B 53, 12621 (1996).

[7] W. Hofstetter and W. Zwerger, Phys. Rev. Lett. 78, 3737 (1997).

[8] K. A. Matveev, Phys. Rev. B 51, 1743 (1995).

[9] K. Flensberg, Phys. Rev. B 48 , 11156 (1993).

[10] Yu. V. Nazarov, Phys. Rev. Lett. 82, 1245 (1999).

[11] D. S. Golubev, A. D. Zaikin, Phys. Rev. Lett. 86, 4887 (2001), A. L. Yeyati et al., Phys. Rev. Lett. 87, 046802 (2001).

[12] M. Kindermann and Yu. V. Nazarov, cond-mat/0304078

[13] D. S. Golubev and A. D. Zaikin, Europhys. Lett 60, 113 (2002).

[14] G. Schön and A. D. Zaikin, Phys. Rep. 198, 237 (1990).

[15] Yu. V. Nazarov and D. A. Bagrets, Phys. Rev. Lett., 88, 196801 (2002).

[16] W. Belzig and Yu. V. Nazarov, Phys. Rev. Lett. 87, 067006 (2001); Phys. Rev. Lett. 87, 197006 (2001).

[17] Yu. V. Nazarov, Ann. Phys. (Leipzig) 8 Spec. Issue, SI193 (1999), cond-mat/9908143

[18] K. A. Matveev, D. Yue, L. I. Glazman, Phys. Rev. Lett. 71, 3351 (1993)

[19] J. Zinn-Justin, Quantum Field Theory and Critical Phenomena (Clarendon Press, Oxford, 1993).

[20] J. M. Kosterlitz, Phys. Rev. Lett 37, 1577 (1976); K. B. Efetov and A. Tschersich, cond-mat/0302257

[21] M. V. Feigelman, A. Kamenev, A. I. Larkin, and M. A. Skvortsov Phys. Rev. B 66, 054502 (2002)

[22] These scales are different in Ref. [13], possibly due to the oversimplified RG they use. The scales were found to be the same in Ref. [21]. 